\title{
Ist die unvollständige Aufklärung von Versuchspersonen ethisch vertretbar?
}

\section{Tanja Krones}

Leitende Ärztin Klinische Ethik, UniversitätsSpital Zürich

Lesen Sie hierzu auch das Interview mit Peter Kleist auf der nächsten Seite.

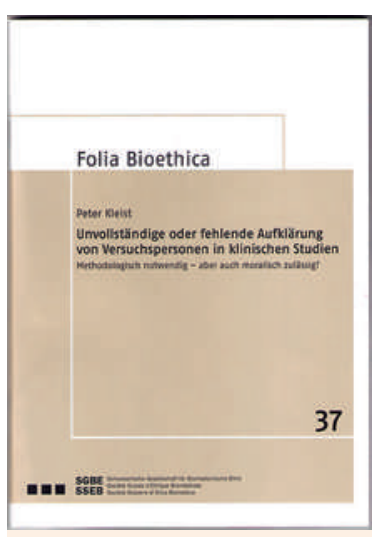

Peter Kleist

Unvollständige oder fehlende Aufklärung von Versuchspersonen in klinischen Studien

Folia Bioethica 37. Basel: Schweizerische Gesellschaft für Biomedizinische Ethik; 2013.

65 Seiten. 15 CHF.

Bestellung:

info[at]bioethics.ch

Korrespondenz:

PD Dr. med. Tanja Krones UniversitätsSpital Zürich DERM C 18

Gloriastrasse 31

CH-8091 Zürich

Tel. 0442553470
Ein weitverbreiteter Glaube, der durchaus auch von manchen Protagonisten im Bereich der Ethik gewinnbringend verkauft wird, besagt, die Ethik sei ein festes Gedankengerüst mit Wahrheitsanspruch und Ewigkeitscharakter, das sich nicht ändern könne (oder dürfe). Ethik ist jedoch nicht von Göttern, sondern von Menschen gemacht, die darum ringen, in schwierigen, uneindeutigen Problemsituationen, die sich im Laufe der Geschichte neu und anders stellen, $\mathrm{zu}$ fairen, bestmöglich begründeten und abgewogenen Lösungen zu kommen. Dazu zählt auch das wichtige Feld der Forschungsethik. Peter Kleist, Facharzt für pharmazeutische Medizin, zurzeit medizinischer Direktor des Pharmaunternehmens GlaxoSmithKline AG in der Schweiz, hat sich seit nun mehr als 20 Jahren mit theoretischen und praktischen Fragen der Forschung und Forschungsethik auseinander- und dafür eingesetzt, dass die (zu Recht umstrittene) pharmazeutische Industrie sich bei allem Eigeninteresse ihrer Verantwortung stellt und sich ernsthaft ethischen Fragen stellt.

Die Forschungsethik-Landschaft ist derzeit sowohl national wie international stark im Umbruch. Neue Forschungsfelder entstehen. Neue politische und fachethische Antworten werden gesucht: In der Schweiz ist das Humanforschungsgesetz auf dem letzten Weg in die Umsetzung. Das Wort von einem Paradigmenwechsel zu einer «personalisierten Medizin» macht die Runde, verbunden mit Versprechen, mittels Biomarkern immer genauere, bessere Medikamente und Therapiekonzepte anbieten zu können. Hierzu bedarf es unter anderem der Sammlung von biologischen und sozialen Kennzeichen von Menschen in internationalen Biobanken über viele Jahre, in denen sich Fragestellungen ergeben können, die bei Sammlung der Daten noch nicht bekannt waren; gleich- einem grossen Problem. Es gibt wissenschaftliche Vorhaben, die nicht durchgeführt, Fragestellungen, die nicht untersucht werden können, wenn Versuchspersonen zuvor umfassend und vollständig aufgeklärt werden. Solche Forschungen wurden und werden (mittlerweile mit weitaus kritischerer Rezeption als noch vor einigen Jahren, das berühmteste Beispiel sind die sogenannten Milgram-Experimente) im Bereich der psychologischen und sozialwissenschaftlichen Forschung häufig durchgeführt. Eine umfassende, vollständige Aufklärung in Forschungsvorhaben ist jedoch ein zentrales Desiderat der am stärksten entwickelten Forschungsethik in der Medizin, die unter anderem in der Deklaration von Helsinki kodifiziert ist. Diese Entwicklung ist wesentlich den vielfältigen bis heute vorkommenden Forschungsskandalen geschuldet, in denen Menschen als Forschungsobjekte missbraucht, getäuscht und zu Schaden gekommen sind. Nimmt man dieses Desiderat jedoch als absolut, kann zum Beispiel nicht methodisch sauber untersucht werden, wie Placebo- und Nocebo-Effekte funktionieren, wie effektiv Präventions-Konzepte in der Versorgung wirken, welche Risikofaktoren gerade bei ärmeren Bevölkerungsschichten, die in Studien eher unterrepräsentiert sind, modifiziert werden können, und wie im Bereich der mittels Biomarkern stratifizierten («personalisierten») Medizin eventuell besser auf Patientengruppen zugeschnittene Therapien gefunden werden können.

Peter Kleist stellt sich in diesem Büchlein, das seine Masterarbeit des Masters of Advanced Studies in Applied Ethics an der Universität Zürich darstellt, ethisch fundiert, anschaulich und mit hochrelevanten allgemeinen forschungsethischen Überlegungen und nachvollziehbaren Schlussfolgerungen diesen

\section{Es gibt wissenschaftliche Vorhaben, die nicht durchgeführt werden können, wenn Versuchspersonen zuvor umfassend und vollständig aufgeklärt werden.}

zeitig steigt das Bewusstsein ökonomischer Verantwortung. Grosse (Heils-)Versprechen lassen sich eher selten halten, Konzepte müssen sich in der Versorgung bewähren. Wir müssen wissen, was tatsächlich etwas bringt; nicht jede Innovation ist gut, weder für das Individuum noch für die Bevölkerung.

Forschungskonzepte, die einige dieser wichtigen Fragen beantworten möchten, stehen jedoch vor
Fragen. Gut begründet kommt er zu dem Schluss, dass es - unter Beachtung zentraler relevanter Kriterien - ethisch vertretbar ist, unter ganz bestimmten Bedingungen Studien zuzulassen, in denen Versuchspersonen nicht vorgängig umfassend aufgeklärt werden. Ein absolut lesenswertes Buch - nicht nur für Ethikkommissionen. 